\title{
Hedgehog signaling controls mouth opening in the amphioxus
}

\author{
Guangwei $\mathrm{Hu}^{1,2,3^{*}}$, Guang $\mathrm{Li}^{3}$ and Yiquan Wang ${ }^{3}$
}

\begin{abstract}
Introduction: The left-sided position of the mouth in amphioxus larvae has fascinated researchers for a long time. Despite the fundamental importance of mouth development in the amphioxus, the molecular regulation of its development is almost unknown. In our previous study, we showed that Hh mutation in the amphioxus leads to no mouth opening, indicating a requirement of Hh signaling for amphioxus mouth formation. Nevertheless, since the Hh mutant also exhibits defects in early left-right (LR) patterning, it remains currently unknown whether the loss of mouth opening is affected directly by Hh deficiency or a secondary effect of its influence on LR establishment.

Results: We demonstrated that knockout of the Smo gene, another key component of the Hh signaling pathway, in the amphioxus resulted in the absence of mouth opening, but caused no effects on LR asymmetry development. Upregulation of Hh signaling led to a dramatic increase in mouth size. The inability of Smo mutation to affect LR development is due to Smo's high maternal expression in amphioxus eggs and cleavage-stage embryos. In Smo mutants, Pou4 and Pax2/5/8 expression at the primordial oral site is not altered before mouth opening.

Conclusions: Based on these results and our previous study, we conclude that Hh signal is necessary for amphioxus mouth formation and that the Hh-mediated regulation of mouth development is specific to the mouth. Our data suggest that Hh signaling regulates mouth formation in the amphioxus in a similar way as that in vertebrates, indicating the conserved role of Hh signaling in mouth formation.
\end{abstract}

Keywords: Hedgehog signal, Mouth opening, Smo, Amphioxus

\section{Background}

Mouth development in animals has fascinated researchers for decades. In most protostomes, the mouth is derived directly from the blastopore. In deuterostomes (including echinoderms, hemichordates and chordates), however, the mouth is thought to develop independently from the blastopore. The first opening formed by the blastopore becomes the organism's anus, while the mouth is formed secondarily on

\footnotetext{
* Correspondence: Hgw0127@163.com

1Jiangsu Key Laboratory of Marine Bioresources and Environment /Jiangsu Key Laboratory of Marine Biotechnology, School of Marine Science and Fisheries, Jiangsu Ocean University, Lianyungang 222005, China

${ }^{2}$ Co-Innovation Center of Jiangsu Marine Bio-industry Technology, Jiangsu Ocean University, Lianyungang 222005, China

Full list of author information is available at the end of the article
}

the opposite side by perforation of the outer epithelium and the wall of the gut.

Among all living deuterostomes, the amphioxus is an exception with respect to mouth formation, in which the mouth initially opens on the left side. Before amphioxus mouth formation, a population of compact mesoderm cells (also called oral mesovesicle, OMV) is present at the posterior end of the first left somite. As development continues, the dorsal group of these cells develops into Hatschek's nephridium, while the ventral group becomes interposed between the ectoderm and endoderm in the region where the mouth will soon form [1, 2]. Mouth penetration occurs between the epidermis and remnant of the OMV $[1,2]$. The peculiar left-sided mouth in the amphioxus is a long-standing conundrum, and much effort has been devoted to homologizing the amphioxus

(c) The Author(s). 2021 Open Access This article is licensed under a Creative Commons Attribution 4.0 International License, which permits use, sharing, adaptation, distribution and reproduction in any medium or format, as long as you give appropriate credit to the original author(s) and the source, provide a link to the Creative Commons licence, and indicate if changes were made. The images or other third party material in this article are included in the article's Creative Commons licence, unless indicated otherwise in a credit line to the material. If material is not included in the article's Creative Commons licence and your intended use is not permitted by statutory regulation or exceeds the permitted use, you will need to obtain permission directly from the copyright holder. To view a copy of this licence, visit http://creativecommons.org/licenses/by/4.0/ The Creative Commons Public Domain Dedication waiver (http://creativecommons.org/publicdomain/zero/1.0/) applies to the data made available in this article, unless otherwise stated in a credit line to the data. 
mouth to that of vertebrates and other deuterostomes [1-9]. Despite the fundamental importance of mouth development in the amphioxus, the molecular mechanisms regulating mouth development in the amphioxus are far less clear. At present, Nodal-Pitx and Bmp signaling pathways have been reported to be associated with mouth formation $[2,10,11]$, and inhibition of Nodal or Bmp signaling results in the loss of left-sided identity, leading to the absence of the mouth, suggesting that these two left-right regulatory pathways do not directly control mouth opening in the amphioxus. A paper by Annona et al. (2017) showed that nitric oxide is an essential cell-signaling molecule for amphioxus mouth formation, which provides the first data for directly revealing a molecular mechanism in amphioxus mouth formation [12]. Nevertheless, it is still necessary to consider other signaling pathways during amphioxus mouth morphogenesis.

The Hedgehog (Hh) signaling pathway is one of the key pathways that is essential for metazoan embryonic development. Its involvement in mouth development has been reported in frog: blocking Hh signaling with the chemical inhibitor cyclopamine or SANT1 resulted in the loss of primary mouth opening [13]. Our previous result showed that Hh loss-of-function resulted in failure of mouth formation in the amphioxus, indicating that the Hh signal may be involved in the regulation of amphioxus mouth development [14]. However, since $\mathrm{Hh}$ deficiency also led to defects in left-right patterning, it remains unclear whether the absence of mouth formation in $H h$ mutants is a direct consequence of Hh perturbation or a secondary effect of impaired left-right patterning.

In this report, we first compared the expression patterns of Smo and Hh genes in the amphioxus Branchiostoma floridae and then investigated whether $\mathrm{Hh}$ signaling in the amphioxus directly controls mouth formation by TALEN-based knockout. Furthermore, we investigated the role of $\mathrm{Hh}$ signaling during amphioxus mouth formation by examining mouth marker genes expression. Together, these findings indicate that $\mathrm{Hh}$ signaling plays a critical role during mouth formation in the amphioxus and points to conservation of this pathway in regulating mouth development.

\section{Material and methods}

\section{Experimental animal}

Amphioxus Branchiostoma floridae were originally acquired from Dr. Jr-Kai Yu (Institute of Cellular and Organismic Biology, Academia Sinica, Taipei, Taiwan), and colonies were maintained in a laboratory culture system as described in the previous report [15]. Thermal induction spawning was performed according to previous report (from $22{ }^{\circ} \mathrm{C}$ to $26^{\circ} \mathrm{C}$ ) [16]. Egg fertilization and embryo culture at $26^{\circ} \mathrm{C}$ were carried out according to previous description [17]. Embryos and larvae at the required developmental stages were fixed with 4\% PFA in MOPS buffer ( $\mathrm{pH}$ 7.4) overnight at $4{ }^{\circ} \mathrm{C}$. All embryos were staged according to previously described methods [18].

\section{Mutant generation and genotyping}

Smo gene knockout amphioxus was generated using the TALEN method. In brief, TALEN pairs recognizing the coding sequence of the Smo gene (Fig. 2a) were designed and assembled according to our previous description [19]. The final TALEN expression plasmids were linearized by SacI restriction enzyme digestion. TALEN mRNA was synthesized using the mMESSAGE mMACHINE T3 kit (Ambion).

TALEN mRNA was microinjected into the egg of the amphioxus followed by fertilization. One day after injection, genomic DNA from injected embryos was isolated and used as the template for PCR. PCR products were digested by the restriction enzyme $\mathrm{BamHI}$ to estimate the somatic mutation ratio. To obtain germline mutations, TALEN-injected embryos (F0 progeny) were raised to adulthood and outcrossed with wild-type amphioxus. Mosaic founder animals were spawned to generate F1 heterozygotes using PCR and sequencing to detect, characterize and follow mutant alleles as described previously [20]. Homozygous mutants were generated by crossing heterozygous animals.

\section{Whole-mount in situ hybridization (WISH)}

The RNA probes used in this study were amplified using the primers listed in Table 1. The cDNA template for PCR was derived from total RNA extracted from mixed embryos and larvae. PCR products were recombined with the pGEM-T Easy vector (Promage, USA) and transformed into E. coli. After sequencing verification, we synthesized digoxigenin DIG-labeled antisense probes for the above genes using SP6 or T7 RNA polymerase (depending on insert orientation). Whole-mount in situ hybridization was performed according to the previously described protocol [21] with slight modifications as follows: the duration of proteinase $\mathrm{K}$ treatment varied from 3 to $10 \mathrm{~min}$ depending on embryonic stage, and probe incubation was performed at $65^{\circ} \mathrm{C}$ overnight.

\section{In vitro mRNA synthesis}

The coding sequence of the $H h$ gene was amplified from a cDNA library based on amphioxus embryos. PCR product was cloned into the pXT7 vector. Plasmid DNA was prepared using Plasmid Mini Kit (Omega), linearized with restriction enzyme, extracted by phenolchloroform and dissolved in RNase-free water. In vitro 
Table 1 List of primers used in this study

\begin{tabular}{|c|c|c|c|}
\hline Name of gene & Forward primer $\left(5^{\prime} \rightarrow 3^{\prime}\right)$ & Reverse primer $\left(5^{\prime} \rightarrow 3^{\prime}\right)$ & Restriction site \\
\hline Smo & GGTACCTTTCCACCATGTTGAGGAGCG & ACTAGTGGTTCTTCACAGTACTCTGTATC & Kpnl/Spel \\
\hline Cer & GGTACCATGAAGACGAGCGTGAGGAGC & ACTAGTTCAGAAGTACTTATCCCCACATG & Kpnl/Spel \\
\hline Nodal & GGTACCGCAGGCCGAGACCAACACCGC & ACTAGTCTACTGACAGCCGCATTCATCC & Kpnl/Spel \\
\hline Lefty & CTCGAGTACGATGAAACCTGTTCTAGTT & $\underline{\text { ACTAGTTTACTGTGTGCACGCACACTG }}$ & Xhol/Spel \\
\hline Pitx & GGTACCACATATCTAAGGAGGACATCGTG & ACTAGTTCTTTAGCAAACAAATCCCATACGC & Kpnl/Spel \\
\hline Ptch & ACGGTTGGACATATTCTGTTGC & TGATACCATCCGCTCATTTCTG & NA \\
\hline Pou4 & GGTACCAGAACAGATGATGAACGGGAAAC & $\underline{\text { ACTAGTTTGGGCGGTGCGATAGTAGAG }}$ & Kpnl/Spel \\
\hline $\operatorname{Pax} 2 / 5 / 8$ & ATGGACAGGATGACCACGATG & GTGAGAAGAGAAGAAGTTGCC & NA \\
\hline Frzb1 & GGTACCGCGATATTGAATTTAGCGTGGT & ACTAGTCGAGTTGTCAGGGTCTTAGCA & Kpnl/Spel \\
\hline
\end{tabular}

mRNA synthesis was conducted using T7 mMESSAGE mMACHINE kit (Ambion).

\section{Results and discussion}

In our previous study, we showed that loss of Hh activity by $H h$ knockout resulted in abnormal left-right patterning and absence of mouth formation in the amphioxus [14]. This raised the possibility that the absence of mouth formation might be a secondary effect of impaired left-right patterning but not directly controlled by Hh signaling. To address this question, we examined the function of Smo in amphioxus mouth formation. Smo is a receptor and a positive regulator of the $\mathrm{Hh}$ signaling pathway in flies and vertebrates [22], and Smo gene knockout would theoretically lead to inactivation of the Hh signaling pathway in the amphioxus. Before performing the Smo mutation experiment, we first analyzed Smo and $H h$ genes expression during several stages of amphioxus embryos with the whole-mount in situ hybridization (WISH) method. We found that Smo exhibited strong maternal expression in fertilized eggs and early cleavage embryos (Fig. 1a1-a4). Zygotic expression of Smo was first detected at the G5 stage in the dorsallateral endomesoderm (Fig. 1a5). In N1 and T1 embryos, Smo was strongly expressed in endomesodermal and neural ectodermal tissues (Fig. 1a7, a8). This result shows that Smo is expressed both maternally and zygotically in amphioxus embryos. This is different from the Hh gene, which shows zygotic but no maternal expression (Fig. 1b1-b8) [23]. Hh expression was first detected at G3 stage. The expression of $H h$ at the L0 stage (the mouth had just opened) was confined to the preoral pit and pharyngeal endoderm (Fig. 1b9, b10). From this, we speculate that zygotic mutation of Smo may not affect the early development of amphioxus embryos (e.g., leftright pattering) but disturbs late developmental events (e.g., mouth formation). We therefore anticipate that if Hh signal regulates mouth formation directly in the amphioxus, loss of $\mathrm{Hh}$ activation by Smo knockout in the amphioxus could lead to the failure of mouth formation. It would, however, not affect the development of left-right asymmetry, which can answer the question we raised at the beginning. To test this hypothesis, we generated Smo gene mutants using the TALEN method as previously described [19].

We constructed two pairs of TALENs targeting the coding sequence of the amphioxus Smo gene, namely, TALEN1 and TALEN2. TALEN mRNAs were injected into amphioxus embryos, and the somatic mutation frequencies were approximately 100\% (TALEN1) and 50\% (TALEN2) (Fig. S1). Because of the high mutant efficiency, TALEN1 mRNA-injected embryos cannot survive to adulthood to generate mosaic F0 animals. In this study, TALEN2 mRNA-injected embryos were raised to adulthood, and one of them carrying mutations at the target site in its germline was crossed with wild-type amphioxus. When F1 progenies developed to adulthood, we identified their genotypes one by one. Heterozygotes $\left(\mathrm{Smo}^{+/-}\right)$with a $10 \mathrm{bp}$ deletion (Fig. 2a) were further used to obtain homozygous mutants. This mutation could induce an open reading frame (ORF) shift and thus generate a truncated protein with no functions. Next, we carefully examined the morphological features of the $\mathrm{Smo}^{-/-}$mutant at the larval stage. Phenotypic examination revealed that the $\mathrm{Smo}^{-/-}$mutants showed curved tails and no mouth opening (Fig. 2c), similar to $H h$ mutants [14]. However, in contrast to $H h$ mutants, Smo mutants developed a normal asymmetric arrangement of pharyngeal organs, including the preoral pit, endostyle and club-shaped gland (Fig. 2c1, c2). To verify that Smo knockout has no effect on left-right patterning in the amphioxus, we examined the expression patterns of left-right regulatory genes (including Cer, Nodal, Lefty and Pitx) in Smo mutants at the N1 neurula stage. At this stage, left-right patterning has already started, and the left-right regulatory genes exhibit an asymmetric expression pattern $[10,20,24-26]$. The results showed that in either wild type, $\mathrm{Smo}^{+/-}$or $\mathrm{Smo}^{-/-}$embryos, Cer was 


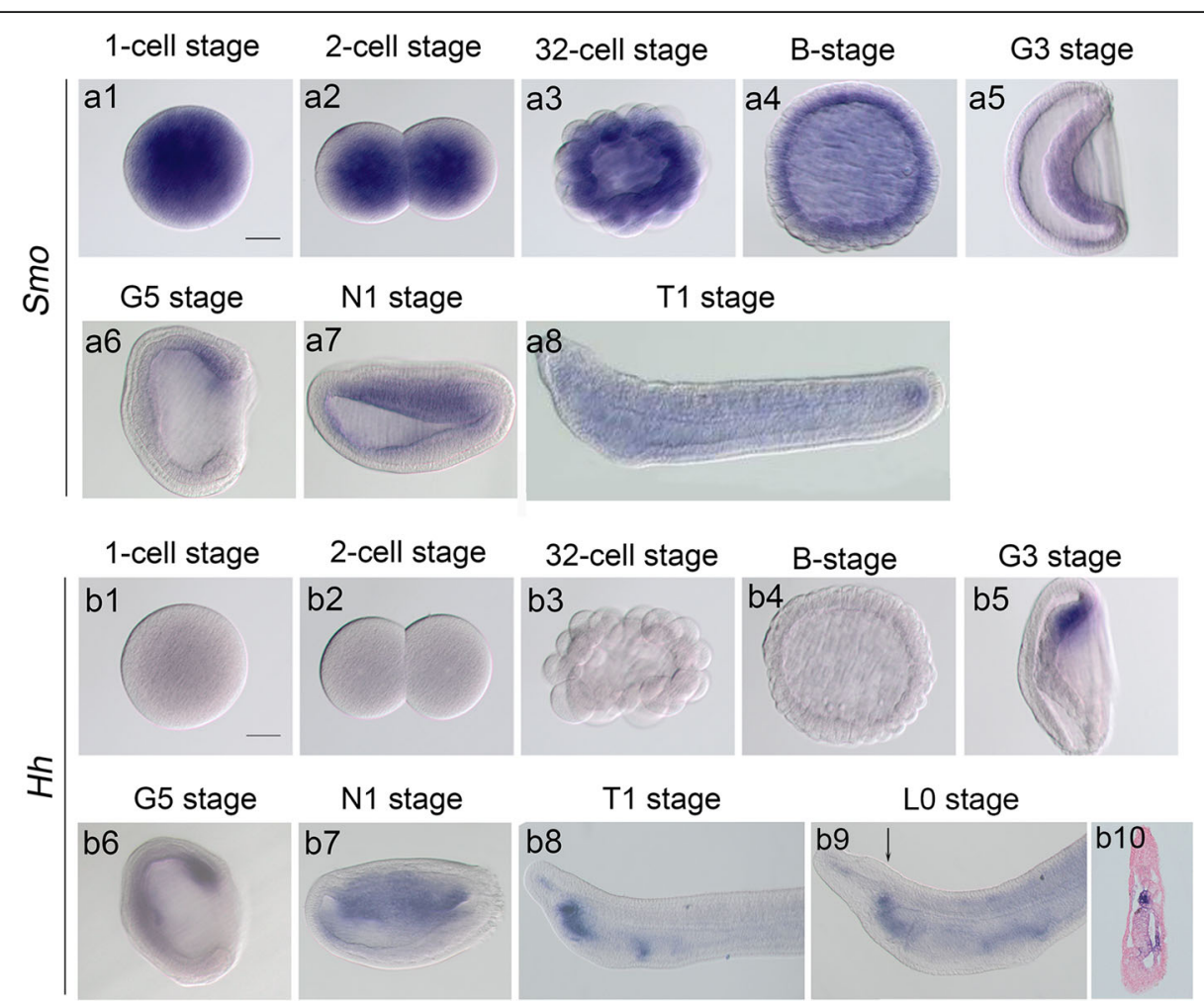

Fig. 1 The expression of Smo and Hh genes in developing amphioxus embryos. a1-a8: Expression patterns of Smo at eight stages of amphioxus development. b1-b10: Expression patterns of Hh at nine stages of amphioxus development. Smo exhibits strong maternal expression in fertilized eggs and early cleavage embryos. Hh gene shows zygotic but no maternal expression. Arrow in b9 indicate the section plane in b10. Scale bar, $50 \mu \mathrm{m}$

expressed on the right side, Nodal exhibited an L $>$ R expression pattern, and Lefty and Pitx were expressed on the left side (Fig. 3). This result indicated that loss of $\mathrm{Hh}$ activation by Smo knockout had no effect on left-right patterning in the amphioxus. From these data and our previous findings [14], we conclude that the Hh signal is necessary for amphioxus mouth formation and that the Hh-mediated regulation of mouth development is specific to the mouth and independent of early morphogenetic defects of abnormal left-right patterning.

To confirm that the inability of Smo knockout to affect left-right patterning is caused by its strong maternal expression, we next examined the expression of Ptch in $\mathrm{Smo}^{-/-}$embryos at the early neurula stage (N1 stage) and tail bud stage (T1 stage) just before mouth opening. Ptch is the direct target of the Hh signaling pathway in vertebrates and the amphioxus [27-29], and thus, its expression can reflect the activity of Hh signaling. The results showed that in wild-type and $\mathrm{Smo}^{+/-}$embryos, Ptch was expressed mainly on the dorsal endoderm at the N1 stage and somatic mesoderm and pharyngeal region at the T1 stage (Fig. 4a, a1). Smo knockout had no effect on Ptch expression at the early neurula stage (Fig. 4a, b) but diminished Ptch expression at the T1 stage (Fig. 4a1, b1). This result indicated that zygotic mutation of Smo had no effect on the activation of Hh signaling at early stage, at least before early neurula stage, showing that the inability of the Smo mutant to affect Hh signaling activation is due to Smo's maternal expression.

In Xenopus, Hh signaling is required to regulate primary mouth size, loss of $\mathrm{Hh}$ activation results in a small or absent primary mouth, and increased Hh activation leads to a larger mouth [11]. To determine whether $\mathrm{Hh}$ signaling regulates mouth size in the amphioxus, we next examined mouth development in $H h$ mRNAinjected embryos. We previously showed that $H h$ mRNA injection effectively upregulates Hh signaling [29]. Compared with control embryos, $H h$ mRNA injection caused a dramatic increase in mouth size (Fig. 5b, b1). Conversely, in Smo-TALEN mRNA-injected (Smo gene knockdown) embryos, 30\% (6/20) showed a small mouth phenotype (Fig. 5c, c1), and Smo homozygous mutants showed a complete loss of mouth opening (Fig. $5 \mathrm{~d}$, d1). Together, these data demonstrate that Hh signaling regulates amphioxus mouth development in a similar way as in vertebrates. 


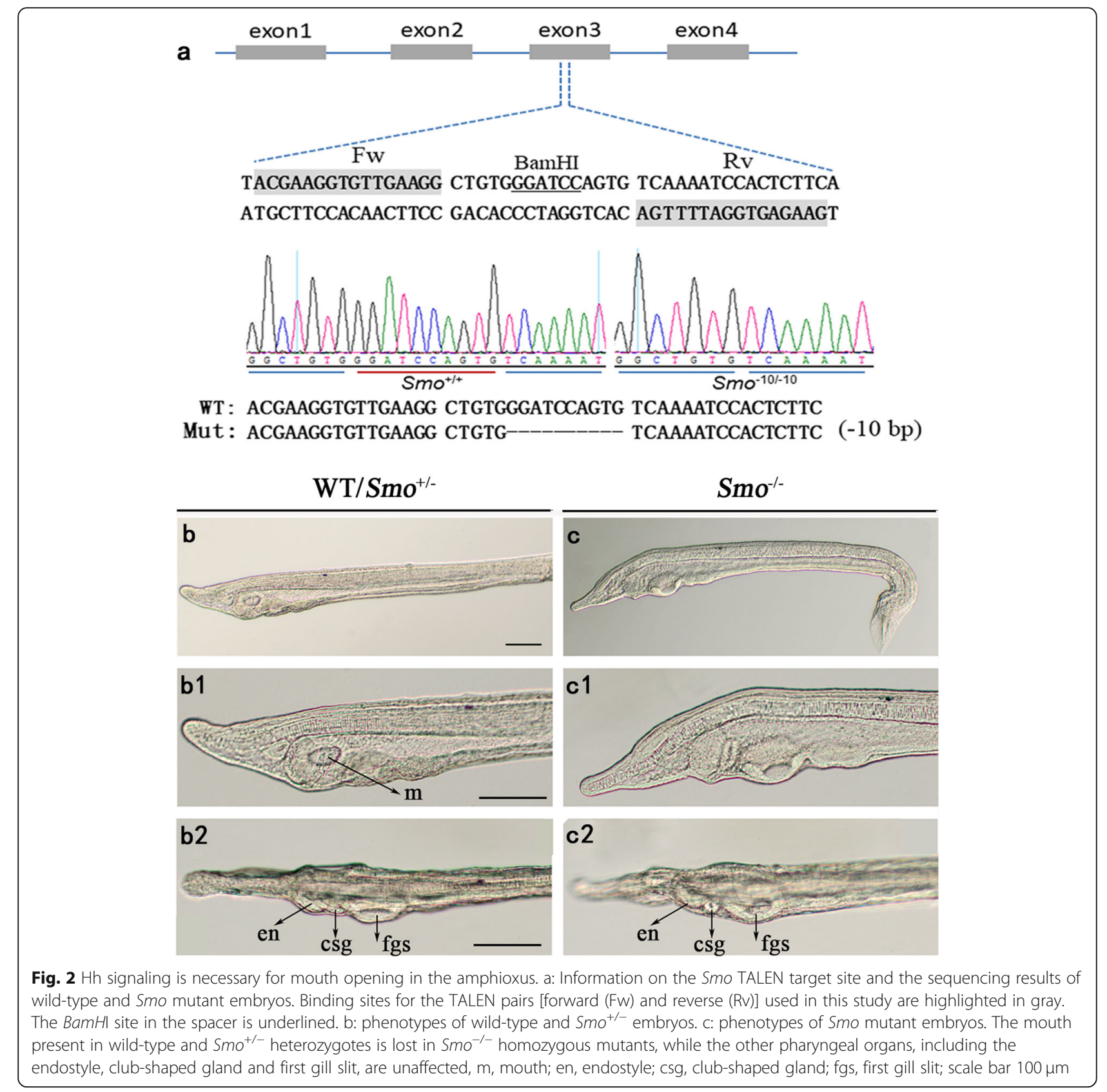

Having shown that the Hh signal is specific to regulate mouth formation and modulate mouth size in the amphioxus, we next tested whether Hh activation functions throughout mouth development or at a specific stage. The developmental process of the amphioxus mouth has been elucidated in previous study, including the formation of OMV and mouth perforation [1, 2], and this process can be visualized by Pou4 expression. Pou4 is expressed dynamically during mouth development: at the early larval stage, Pou4 is expressed in the oral region, including the $\mathrm{OMV}$, and then at the margin of the mouth during perforation [30]. To determine the functional stage of $\mathrm{Hh}$ signaling during amphioxus mouth development, we examined the expression of Pou4 in Smo mutant embryos. The results showed that in wild-type embryos, Pou4 was expressed in the primordial oral site before mouth opening and then at the margin of the mouth after the mouth had opened (Fig. 6a, b). Loss of Hh activation by Smo gene knockout did not affect Pou4 expression at the T1 stage (before mouth opening) (Fig. 6a1) but diminished the expression of Pou4 at the margin of the mouth with the complete loss 

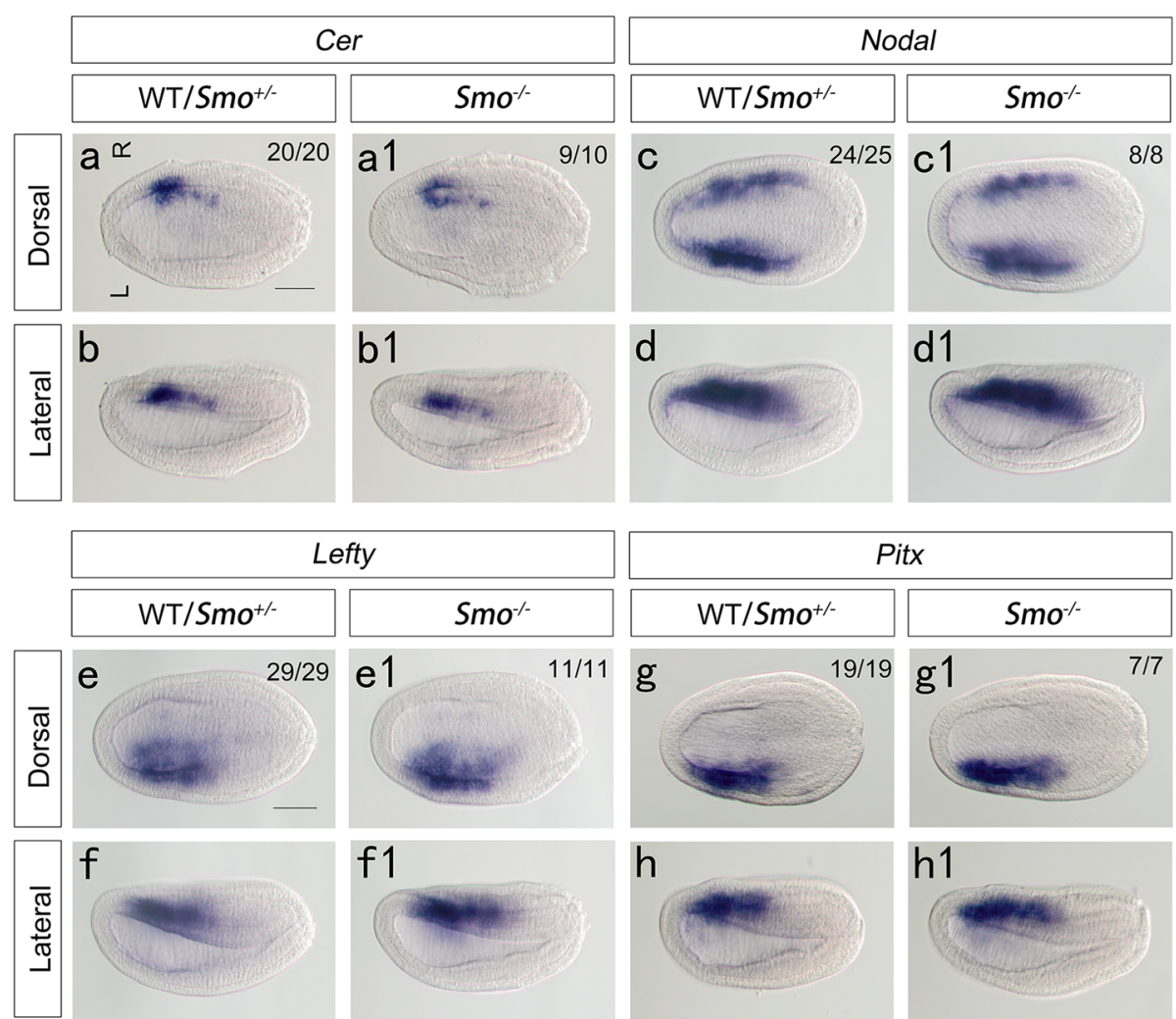

f1

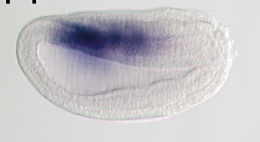

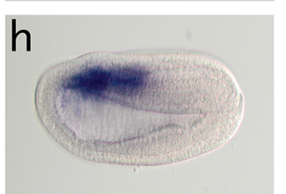

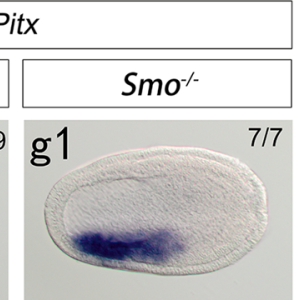

\section{h1}

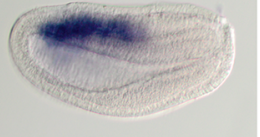

Fig. 3 Expression of left-right regulatory genes in Smo mutant embryos. a, b: In the wild-type and Smo ${ }^{+/-}$embryos, Cer is expressed mainly in the right paraxial mesoderm. a1, b1: Cer expression is unaffected in Smo mutant embryos. c, d: Nodal exhibits an L $>$ R pattern in WT/Smo ${ }^{+/-}$amphioxus at the early neurula stage. $\mathrm{cl}$, d1: The asymmetrical expression of Nodal is unaffected in Smo mutant embryos. e, f: Lefty is expressed on the left side in

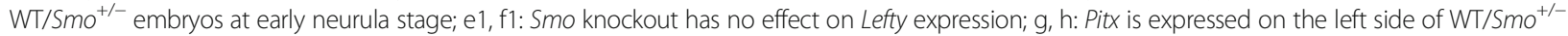
embryos, Smo knockout has no effect on Pitx expression. Anterior to the left; L, left side; R, right side; Scale bar, 50 um; Numbers in the top right corner of a panel show the number of times the phenotype depicted was seen out of the total number of embryos from that genotype analyzed

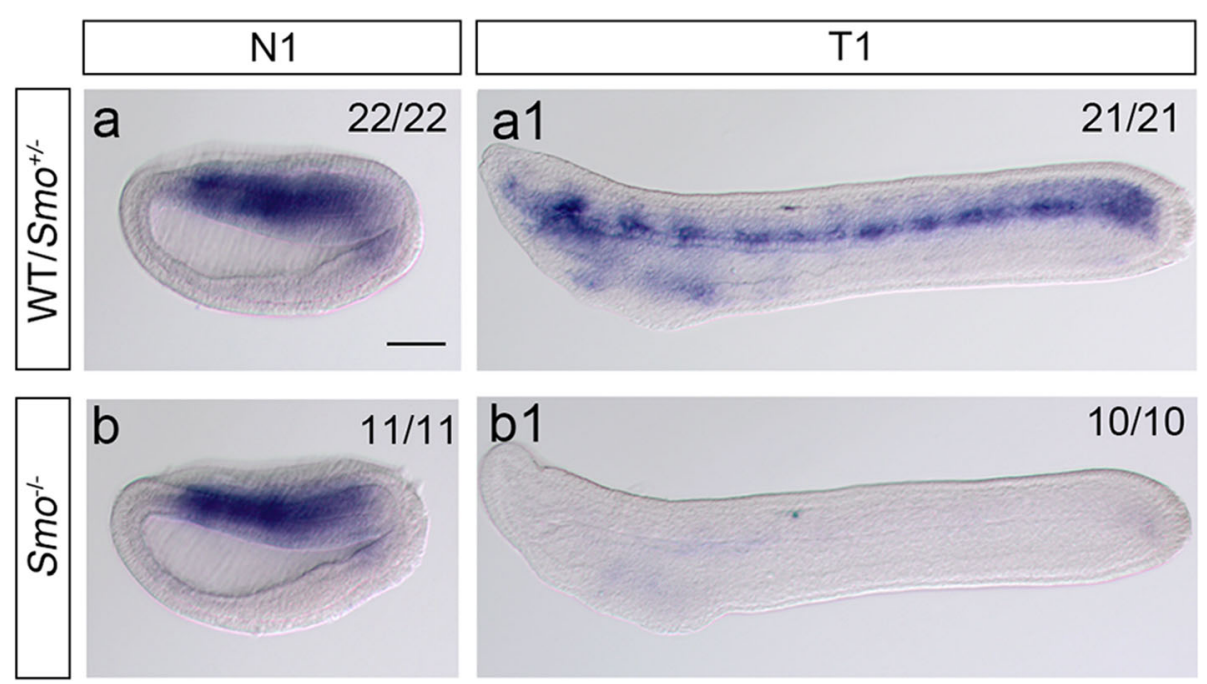

Fig. 4 Ptch expression in Smo mutant embryos. Ptch expression was visualized by in situ hybridization, and all embryos were placed anterior to the left; a, b: Ptch expression in WT or Smo ${ }^{+/-}$embryos; a1, b1: Ptch expression in Smo ${ }^{-/-}$embryos. Smo gene knockout has no effect on Ptch expression at the $\mathrm{N} 1$ stage but results in diminished Ptch expression at the $\mathrm{T} 1$ stage. N1, early neurula stage; $\mathrm{T} 1$, tail bud stage before mouth opening; scale bar, $100 \mu \mathrm{m}$ 

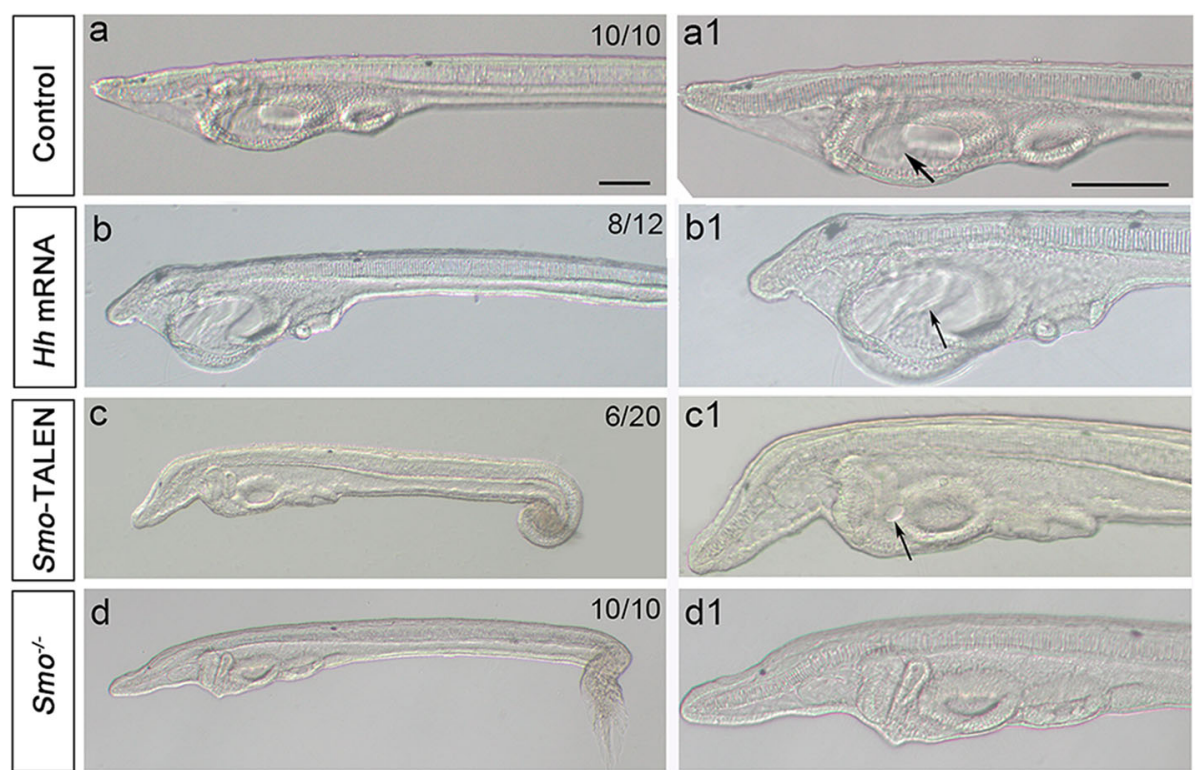

Fig. 5 Hedgehog perturbation affects the size of amphioxus mouth. a, a1: Left lateral view of L1 stage larvae focused on the left-sided mouth opening in control embryos (black arrow), Scale bar, $100 \mu \mathrm{m}$. b, b1:Hh mRNA injection results in a dramatic increase in mouth size. c, c1: SmoTALEN mRNA injection results in a small mouth in the amphioxus. $d$, $d 1$ : Smo gene knockout leads to a complete loss of mouth opening. Numbers in the top right corner of a panel show the number of times the phenotype was observed in the total number of embryos examined

of mouth opening (Fig. 6b1). This result showed that the initial specification of the mouth in the amphioxus may not depend on Hh signaling. To verify this, we also examined the expression of Pax $2 / 5 / 8$ at the primordial oral site before mouth opening (T1 stage) [31]. In agreement with Pou4 expression at the T1 stage, Pax2/5/8 expression was not affected at the T1 stage in embryos with loss of Hh activation (Fig. S2). Taken together, these results indicated that the initial specification of the mouth may not depend on Hh activation; however, the perforation of the mouth is probably controlled by $\mathrm{Hh}$ signaling.

In vertebrates, oral perforation is characterized by dissolution of the ectoderm and endoderm, and Hh signaling plays a key role in this process [13]. In the amphioxus, the molecular mechanisms regulating mouth formation remain rather scarce, especially for perforation. In this study, we showed that Hh signaling is necessary for the development of the mouth in amphioxus larvae probably through controlling perforation. Until

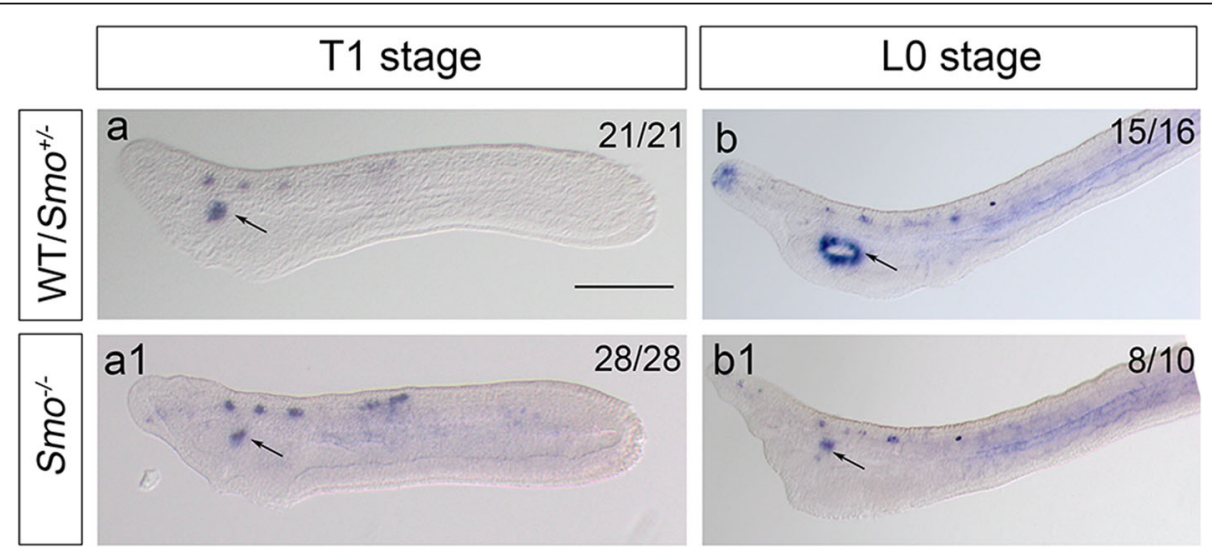

Fig. 6 Pou4 expression during mouth development in the amphioxus. Images of B. floridae at the tail bud stage (T1 stage) and the open mouth stage (L0 stage) from the left lateral view. Black arrows mark the mouth region, scale bar, $100 \mu \mathrm{m}$. a, a1: Pou4 is expressed focally in the regions destined to form the mouth (a), Smo gene knockout has no effect on Pou4 expression (a1). b, b1: Pou4 is expressed at the margin of the mouth at the L0 stage. Smo knockout results in diminished Pou4 expression at the mouth margin with the complete loss of mouth opening 
now, it has been uncertain whether amphioxus mouth penetration results from fusion of the ectoderm and endoderm like that in vertebrates [1]. A Study in Xenopus showed that Wnt antagonists Frzb1 and Crescent regulate mouth perforation in the developing primary mouth [32]. During primary mouth formation, Frzb1 and Crescent inhibit Wnt signaling, which prevents the synthesis of the proteins Laminin and Fibronectin, which are essential for basement membrane dissolution [32]. Although no basal lamia around the OMV was found during amphioxus mouth formation [2], we showed that loss of Hh activation diminished Frzb1 expression in the mouth region (Fig. S3). Therefore, it is tempting to hypothesize that Wnt signaling may exert important roles during amphioxus mouth perforation, similar to that in vertebrates. Further studies are needed to investigate the relationship of $\mathrm{Hh}$ and Wnt pathway during amphioxus mouth perforation and to clarify whether the amphioxus mouth is homologous to that of vertebrates.

\section{Conclusion}

In this study, we showed that Smo is expressed maternally and zygotically in $B$. floridae, which is different from the previous report that Smo expression begins at the blastula stage in Branchiostoma belcheri [33]. Thanks to the maternal expression of Smo, loss of Hh activation by Smo knockout did not affect amphioxus left-right asymmetric development but resulted in a complete loss of mouth formation, showing that Hhmediated regulation of mouth development is specific to the mouth and can be uncoupled from early defects of impaired left-right patterning. Our results provide the first demonstration of a role for Hh signaling in amphioxus mouth development. The unusual location of the amphioxus mouth has puzzled researchers for more than 100 years, and various hypotheses have been proposed to explain the evolutionary history of the amphioxus mouth. Our results significantly advance our understanding of amphioxus mouth development and provide a new direction for researchers to further explore the genetic regulation of mouth development. Moreover, our results pinpoint a novel role for Hh signaling during amphioxus embryo development.

\section{Abbreviations}

Hh: Hedgehog; LR: Left-right; Smo: Smoothened; OMV: Oral mesovesicle; Ptch: Patched

\section{Supplementary Information}

The online version contains supplementary material available at https://doi. org/10.1186/s40851-021-00186-8.

Additional file 1: Fig. S1 TALEN-mediated genome editing at amphioxus Smo locus. a: FO Mutagenesis efficiency of two Smo-TALEN targets (estimated as percentages of uncut PCR products), M: DNA marker, WT shows PCR products amplified from the genomic DNA extracted from wild-type embryos and treated with restriction endonucleases; TALEN1 shows the efficiency of target 1, TALEN2 shows the efficiency of target 2 (used in this study). White arrows mark the uncut bands; $\mathbf{b}$ : Mutagenesis efficiency of FO (1\# male) generation gamete, PCR: PCR product without enzyme digestion; c: Sampling test of F1 generation, numbers indicate the number of individuals. Number 2 is heterozygote

Additional file 2: Fig. S2 Pax2/5/8 expression in Smo mutant embryos. Pax2/5/8 expression was visualized by in situ hybridization. All embryos were placed with the head to the left, arrows mark the regions destined to form the mouth, scale bar, $100 \mu \mathrm{m}$; a, left lateral view; b, dorsal view. Smo knockout has no effect on Pax2/5/8 expression at the region where the mouth will form.

Additional file 3: Fig. $\mathbf{S 3} \mathrm{Frzb}_{1}$ expression in Hh mutant embryos. Frzb expression at the $\mathrm{LO}$ stage was visualized by in situ hybridization. All embryos were placed with the head to the left, and the arrow marks the mouth region. Scale bar, $100 \mu \mathrm{m}$. Loss of Hh activation diminished Frzb1 expression at the mouth region.

\section{Acknowledgments}

We would like to thank Xian Liu for her technical support with whole-mount in situ hybridization. We also thank Nanjing Ji for linguistic corrections.

\section{Authors' contributions}

$\mathrm{GH}$ and $\mathrm{GL}$ carried out all of the experiments. GH, GL, and YW designed the study and wrote the manuscript. All authors read and approved the final manuscript.

\section{Funding}

This work was supported by grants from the National Natural Science Foundation of China (No. 31900370), the Natural Science Foundation of Jiangsu Province (No. BK20191007), the Priority Academic Program Development Fund of Jiangsu Higher Education Institutions (PAPD), the Open Research Fund of Jiangsu Key Laboratory of Marine Biotechnology (HS2019001), and Lianyungang 521 Talent Projects, No. 2021-1021.

\section{Availability of data and materials}

The datasets used and/or analyzed during the current study are available from the corresponding author on reasonable request.

\section{Declarations}

Ethics approval and consent to participate

Not applicable.

Consent for publication

Not applicable.

Competing interests

The authors declare that they have no competing interests.

\section{Author details}

'Jiangsu Key Laboratory of Marine Bioresources and Environment /Jiangsu Key Laboratory of Marine Biotechnology, School of Marine Science and Fisheries, Jiangsu Ocean University, Lianyungang 222005, China.

${ }^{2}$ Co-Innovation Center of Jiangsu Marine Bio-industry Technology, Jiangsu Ocean University, Lianyungang 222005, China. ${ }^{3}$ State Key laboratory of

Cellular Stress Biology, School of Life Sciences, Xiamen University, Xiamen 361102, China.

Received: 11 June 2021 Accepted: 8 October 2021

Published online: 24 December 2021

\section{References}

1. Holland ND. Formation of the initial kidney and mouth opening in larval amphioxus studied with serial blockface scanning electron microscopy (SBSEM). Evodevo. 2018:9(1):1-16. https://doi.org/10.1186/s13227-018-01 04-3. 
2. Kaji T, Reimer JD, Morov AR, Kuratani S, Yasui K. Amphioxus mouth after dorso-ventral inversion. Zool Lett. 2016;2(1):1-14. https://doi.org/10.1186/s4 0851-016-0038-3.

3. Legros R. Sur quelque points de l'anatomie et du développement de I'Amphioxus. Anat Anz. 1910;35(561):87.

4. MacBride EW. Memoirs: the formation of the layers in amphioxus and its bearing on the interpretation of the early ontogenetic processes in other vertebrates. J Cell Sci. 1909;2(215):279-345. https://doi.org/10.1242/jcs.s254.215.279.

5. Medawar PB. Asymmetry of larval Amphioxus. Nature. 1951;167(4256):852-3. https://doi.org/10.1038/167852a0.

6. Ruppert EE. Morphology of Hatschek's nephridium in larval and juvenile stages of Branchiostoma virginiae (Cephalochordata). Israel J Zool. 1996; 42(Suppl):161-82.

7. Soukup V, Kozmik Z. Zoology: a new mouth for amphioxus. Curr Biol. 2016; 26(9):R367-R8. https://doi.org/10.1016/j.cub.2016.03.016

8. van Wijhe JW. Beiträge zur Anatomie der Kopfregion des Amphioxus lanceolatus. Petrus Camper. 1901;1:109-15

9. Yasui K, Kaji T. The lancelet and ammocoete mouths. Zool Sci. 2008;25(10): 1012-9. https://doi.org/10.2108/zsj.25.1012.

10. Soukup V, Yong LW, Lu T-M, Huang S-W, Kozmik Z, Yu J-K. The nodal signaling pathway controls left-right asymmetric development in amphioxus. EvoDevo. 2015:6(1):1-23. https://doi.org/10.1186/2041-9139-6-5.

11. Soukup V, Kozmik Z. The bmp signaling pathway regulates development of left-right asymmetry in amphioxus. Dev Biol. 2018;434(1):164-74. https://doi. org/10.1016/j.ydbio.2017.12.004.

12. Annona G, Caccavale F, Pascual-Anaya J, Kuratani S, De Luca P, Palumbo A, et al. Nitric oxide regulates mouth development in amphioxus. Sci Rep. 2017;7(1):8432. https://doi.org/10.1038/s41598-017-08157-w

13. Tabler JM, Bolger TG, Wallingford J, Liu KJ. Hedgehog activity controls opening of the primary mouth. Dev Biol. 2014;396(1):1-7. https://doi.org/1 0.1016/j.ydbio.2014.09.029.

14. Hu G, Li G, Wang H, Wang Y. Hedgehog participates in the establishment of left-right asymmetry during amphioxus development by controlling Cerberus expression. Development. 2017;144(24):4694-703. https://doi.org/1 $0.1242 /$ dev. 157172

15. Li G, Yang X, Shu Z, Chen X, Wang Y. Consecutive spawnings of Chinese amphioxus, Branchiostoma belcheri, in captivity. PLoS One. 2012;7(12): e50838. https://doi.org/10.1371/journal.pone.0050838.

16. Li G, Shu Z, Wang Y. Year-round reproduction and induced spawning of Chinese amphioxus, Branchiostoma belcheri, in laboratory. PLoS One. 2013; 8(9):e75461. https://doi.org/10.1371/journal.pone.0075461.

17. Zhang QJ, Sun Y, Zhong J, Li G, Lü XM, Wang YQ. Continuous culture of two lancelets and production of the second filial generations in the laboratory. J Exp Zool B Mol Dev Evol. 2007;308(4):464-72. https://doi.org/1 0.1002/jez.b.21172

18. Carvalho JE, Lahaye F, Yong LW, Croce JC, Escrivá H, Yu J-K, et al. An updated staging system for cephalochordate development: one table suits them all. Front Cell Dev Biol. 2021;9:668006. https://doi.org/10.3389/fcell.2 021.668006

19. Li G, Feng J, Lei Y, Wang J, Wang H, Shang L-K, et al. Mutagenesis at specific genomic loci of amphioxus Branchiostoma belcheri using TALEN method. J Genet Genomics. 2014;41(4):215-9. https://doi.org/10.1016/j.jgg.2 014.02.003.

20. Li G, Liu X, Xing C, Zhang H, Shimeld SM, Wang Y. Cerberus-nodal-lefty-Pitx signaling cascade controls left-right asymmetry in amphioxus. Proc Natl Acad Sci U S A. 2017;114(14):3684-9. https://doi.org/10.1073/pnas.162051 9114.

21. Holland LZ, Holland PWH, Holland ND, Ferraris JD, Palumbi SR. Revealing homologies between body parts of distantly related animals by in situ hybridization to developmental genes: amphioxus versus vertebrates. In: Ferraris JD, Palumbi SR, editors. Molecular zoology: advances, strategies, and protocols. New York: Wiley-Liss; 1996. p. 267-82.

22. Varjosalo M, Taipale J. Hedgehog signaling. J Cell Sci. 2007;120(1):3-6. https://doi.org/10.1242/jcs.03309.

23. Shimeld SM. The evolution of the hedgehog gene family in chordates: insights from amphioxus hedgehog. Dev Genes Evol. 1999;209(1):40-7 https://doi.org/10.1007/s004270050225.

24. Le Petillon Y, Oulion S, Escande M-L, Escriva H, Bertrand S. Identification and expression analysis of BMP signaling inhibitors genes of the DAN family in amphioxus. Gene Expr Patterns. 2013;13(8):377-83. https://doi.org/10.1016/j. gep.2013.07.005.

25. Yasui K. Zhang S-C, Uemura M, Saiga $H$. left-right asymmetric expression of BbPtx, a Ptx-related gene, in a lancelet species and the developmental leftsidedness in deuterostomes. Development. 2000;127(1):187-95. https://doi. org/10.1242/dev.127.1.187

26. Yu JK, Holland LZ, Holland ND. An amphioxus nodal gene (AmphiNodal) with early symmetrical expression in the organizer and mesoderm and later asymmetrical expression associated with left-right axis formation. Evol Dev. 2002;4(6):418-25. https://doi.org/10.1046/j.1525-142X.2002.02030.X.

27. Hooper JE, Scott MP. Communicating with hedgehogs. Nat Rev Mol Cell Bio. 2005;6(4):306-17. https://doi.org/10.1038/nrm1622.

28. Lee J, Platt KA, Censullo P, i Altaba AR. Gli1 is a target of sonic hedgehog that induces ventral neural tube development. Development. 1997;124(13): 2537-52. https://doi.org/10.1242/dev.124.13.2537.

29. Zhu X, Shi C, Zhong Y, Liu X, Yan Q, Wu X, et al. Cilia-driven asymmetric Hedgehog signalling determines the amphioxus left-right axis by controlling Dand5 expression. Development. 2020;147(1):dev182469.

30. Candiani S, Oliveri D, Parodi M, Bertini E, Pestarino M. Expression of AmphiPOU-IV in the developing neural tube and epidermal sensory neural precursors in amphioxus supports a conserved role of class IV POU genes in the sensory cells development. Dev Genes Evol. 2006:216(10):623-33. https://doi.org/10.1007/s00427-006-0083-6.

31. Kozmik Z, Holland ND, Kalousova A, Paces J, Schubert M, Holland LZ Characterization of an amphioxus paired box gene, AmphiPax2/5/8: developmental expression patterns in optic support cells, nephridium, thyroid-like structures and pharyngeal gill slits, but not in the midbrainhindbrain boundary region. Development. 1999;126(6):1295-304. https://doi. org/10.1242/dev.126.6.1295.

32. Dickinson AJ, Sive HL. The Wnt antagonists Frzb-1 and crescent locally regulate basement membrane dissolution in the developing primary mouth. Development. 2009;136(7):1071-81. https://doi.org/10.1242/dev.032 912.

33. Lin Y, Cai Z, Huang S, Yang L, Wang C, Liu Z, et al. Ptc, Smo, Sufu, and the hedgehog signaling pathway in amphioxus. Evol Dev. 2009;11(6):710-8. https://doi.org/10.1111/j.1525-142X.2009.00378.x

\section{Publisher's Note}

Springer Nature remains neutral with regard to jurisdictional claims in published maps and institutional affiliations.

\section{Ready to submit your research? Choose BMC and benefit from:}

- fast, convenient online submission

- thorough peer review by experienced researchers in your field

- rapid publication on acceptance

- support for research data, including large and complex data types

- gold Open Access which fosters wider collaboration and increased citations

- maximum visibility for your research: over $100 \mathrm{M}$ website views per year

At $\mathrm{BMC}$, research is always in progress.

Learn more biomedcentral.com/submission 